\title{
Daily rhythms in hormonal markers of diabetes and obesity: effect of weight and Type 2 diabetes
}

\author{
Cheryl Isherwood, Daniella T. Otway, Simone Mäntele, Benita Middleton, John Wright, \\ M. Denise Robertson, Debra J. Skene, Michelle Gibbs and Jonathan D. Johnston \\ Faculty of Health and Medical Sciences, University of Surrey, Guildford, GU2 7XH, UK
}

Concentrations of some circulating hormones associated with energy metabolism and homeostasis are under the influence of the 24 hour circadian timing system, controlled by the central oscillator in the suprachiasmatic nuclei (SCN) of the hypothalamus and peripheral clocks, including those in endocrine organs along the alimentary canal and in adipose tissue. Circulating concentrations of these hormones are also influenced by feeding, weight and type 2 diabetes mellitus (T2DM). To date, however, 24-hour rhythmicity has only been investigated for a small number of relevant hormones.

The aim of this study was to investigate the effect of weight and T2DM on the concentration and rhythmicity of these hormones across a $24 \mathrm{~h}$ day under highly controlled laboratory conditions.

Men aged between 45-65 years were recruited into lean $(n=8)$, overweight $(\mathrm{OW})(n=10)$ or OW with T2DM $(n=7)$ groups ${ }^{(1)}$. They followed a strict sleep-wake and diet regime for 1 week before the laboratory session. During the laboratory session, they were kept in a controlled light-dark environment, remained semi-recumbent throughout and received identical hourly Fortisip meal replacement milkshakes calculated at 1.5 times BMR from $15: 30 \mathrm{~h}$ on day 1 to $06: 30 \mathrm{~h}$ on day 3 during waking hours $(06: 30 \mathrm{~h}-22: 30 \mathrm{~h})$. Hourly blood samples were taken from 07:00 h on day 2 until 07:00 h on day 3, collected in di-potassium EDTA-coated tubes. Plasma C-peptide, insulin, ghrelin, leptin, GIP, GLP-1, glucagon, PAI-1, resistin and visfatin were measured using the Bio-Plex Pro ${ }^{\text {TM }}$ Diabetes Assay. The table below compares average hormone concentrations across the 24-hour period in the 3 groups.

\begin{tabular}{|c|c|c|c|c|c|c|c|c|c|}
\hline & \multicolumn{2}{|l|}{ Lean } & \multicolumn{2}{|l|}{ OW } & \multicolumn{2}{|l|}{ T2DM } & \multicolumn{3}{|l|}{$P$ value } \\
\hline & mean $\mathrm{pg} / \mathrm{ml}$ & SEM & mean $\mathrm{pg} / \mathrm{ml}$ & $\overline{\text { SEM }}$ & mean $\mathrm{pg} / \mathrm{ml}$ & SEM & lean: OW & lean: T2DM & OW: T2DM \\
\hline C-peptide & 1319 & 204 & 1624 & 81 & 1811 & 118 & $0 \cdot 004$ & $<0.001$ & \\
\hline Insulin & 491 & 58 & 1122 & 105 & 1487 & 214 & $<0.001$ & $<0.001$ & $<0.001$ \\
\hline Ghrelin & 1087 & 159 & 1316 & 110 & 1107 & 71 & $<0 \cdot 001$ & & $<0 \cdot 001$ \\
\hline Leptin & 1433 & 286 & 8533 & 1602 & 7871 & 1730 & $<0.001$ & $<0.001$ & \\
\hline GIP & 709 & 94 & 1044 & 120 & 1295 & 410 & $0 \cdot 023$ & $<0.001$ & \\
\hline GLP-1 & 223 & 46 & 338 & 31 & 362 & 32 & $<0 \cdot 001$ & $<0 \cdot 001$ & \\
\hline glucagon & 269 & 16 & 450 & 14 & 437 & 27 & $<0.001$ & $<0.001$ & \\
\hline PAI-1 & 5064 & 556 & 8671 & 772 & 5571 & 351 & $<0 \cdot 001$ & & $<0 \cdot 001$ \\
\hline Resistin & 3104 & 263 & 3697 & 302 & 3859 & 477 & & $0 \cdot 016$ & \\
\hline visfatin & 7669 & 1184 & 7408 & 1004 & 26360 & 15139 & & $<0 \cdot 001$ & $<0 \cdot 001$ \\
\hline
\end{tabular}

$P$ value shows the significant difference between groups (one way ANOVA followed by post hoc Tukey HSD)

To determine daily rhythmicity, each data point was expressed as a percentage of the 24-h mean for each analyte. Cosinor analysis was then performed on the grouped data to assess the fit of a cosine curve. The results indicated significant $(p<0 \cdot 05)$ rhythmicity for C-peptide, insulin, ghrelin, leptin, GIP and GLP-1 in one or more groups, with C-peptide, insulin and leptin showing a diurnal rhythm in all 3 groups. Ghrelin displayed a clear diurnal rhythm in the lean group but not in OW and T2DM. As expected, concentrations of C-peptide, insulin, leptin, GIP, GLP-1, glucagon, were significantly increased in the OW and T2DM groups (see table).

In conclusion, our data show plasma concentrations were significantly different in the OW and T2DM groups compared with the lean group in 6 of the 10 analytes. Some analytes (6 of the 10) exhibited daily rhythmicity in one or more of the groups.

This work was funded by Diabetes-UK (grant 08/0003607), EU FP7-HEALTH-2011 EuRhythDia (grant 278397), BBSRC (grant BB/I019405/1), BBSRC (grant BB/D526853/1) and Stockgrand Ltd.

1. Mäntele S, Otway DT, Middleton B, et al. (2012) PLoS ONE 7(5): e3. 